\section{Childhood Goitre.}

It was my intention, had time permitted, to refer in some detail to childhood goitre, since many of the observations hitherto recorded in regard to goitre prevention by iodine relate to the thyroid swellings of childhood. I must, however, content myself with the mention of a few points. Many of these swellings in children are physiological hypertrophies often normal to the individual, and have no title to be classed as "goitre" - a term which signifies thyroid disease. The great majority of them-as many as 98 per cent. (Olin) - are slight, some so slight that but a fleeting glimpse of the gland's outlines are caught as the child swallows. If all these be classed as goitre a wholly erroneous idea of the prevalence of thyroid disease may be gained. Further, estimates as to the prevalence of goitre may vary within wide limits according to what the observer may regard as a goitre. Thus, in a school where goitre was endemic; I have known the malady suddenly to assume epidemic proportions with the advent of one school medical officer, and the epidemic to disappear as suddenly with the advent of another.

A small minority of thyroid swellings in children are true goitres; in Himalayan India these goitres are conspicuous, often unilateral and right-sided, but sometimes regular and bilateral enlargements of the gland, which not infrequently are associated with engorgement of the veins of the neck; they may persist through puberty into adult life and begin to show adenomatous nodules in their substance as early as the twelfth year. They are as obviously true "goitres" as similar swellings in older subjects. "Childhood goitres" attain to their highest incidence in boys at the age of 9 and in girls at the age of 10 in Himalayan India; but the peaks of highest incidence appear to differ in different localities and appear also to be related to the prevailing type of goitre. Thus, where colloid goitre is the prevalent type, childhood goitre is said to be at its highest incidence in boys about the age of 8 to 10 , and in girls about the age of 12. The incidence of thyroid enlargement in children below the age of puberty is not of itself a true index of the endemicity of goitre; its incidence may be low and yet the endemicity of adeno-parenchymatous goitre be high, or the incidence of childhood goitre may be high and yet goitre may not be a conspicuous feature in the adult population.

The incidence of childhood goitre is only of value as evidence of prevailing thyroid disease when considered in relation to the other criteria of endemicity and to the prevalence of goitre in the adult population. A marked characteristic of the thyroid swellings of childhood is their tendency to spontaneous disappearance. This tendency varies in different localities, and is probably related to the prevailing type of goitre; in endemic foci in Himalayan India I have found 11 per cent. of these swellings to disappear spontaneously in boys and 9 per cent. in girls; Nicholson has placed the figure as high as 50 per cent. in a certain endemic area in the Himalayan foot-hills; while Bircher reports that although 70 to 100 per cent. of children may be the subjects of swollen thyroids in certain parts of Switzerland, not more than 3 to 9 per cent. of young men are rejected because of goitre when they present themselves for military service. It is necessary to bear these facts in mind, not only when inaugurating iodine prophylaxis in any locality, but when attempting to form an estimate of its value.

\section{Conclusion}

I have endeavoured to indicate the broad lines on which we must, in my opinion, view diseases of the thyroid gland. It is, I believe, rare that any metabolic disorder such as goitre is due to a single cause. There is, as a rule, a multiplicity of factors involved. The thyroid gland cannot be considered apart from the rest of the body, nor iodine apart from other food constituents. The time has come when we must search more closely for causes of the thyroid gland's disorders in disturbances at the threshold of absorption in the intestine, in disturbances at the threshold of utilization in the tissues, and in disturbances in the cell and tissue plasma of the gland itself.

\section{An Adromas}

ox

\section{DUODENAL AND GASTRIC ULCERATION : SOME PERSONAL EXPERIENCES.* \\ nY}

T. P. DUNHILL, C.M.G., M.D., ASSISTANT DIRECTOR, SURGICAL PROFESSORIAL UNIT, ST. BARTHOLOMEW'S HOSPITAL.

(With Special Plate.)

I Have chosen this subject for two reasons. In the first place, whether we be general practitioners, physicians, or surgeons, its problems are constantly coming before us, and have to be dealt with. No one can believe that the methods of treatment are so standardized that the right and only thing to be done in each instance is settled, and we have all lived long enough to know that what we have done has not invariably turned out to be the best. Men of large experience have written and spoken extensively on this subject, and I make no pretence of saying the final word on this occasion; but we each in our different capacities have to accept responsibility towards those patients who come to us for advice. In the second place, I have for some years tried, in conjunction with my colleagues, to attain the maximum degree of accuracy in diagnosis of gastric and duodenal ulcers by means of $x$-ray examination. The skiagrams are typical of the results that are to be expected whenever a patient is well enough to have the examination made.

I shall not attempt to cover all the ground. This short address must perforce be discursive. In any surgeon's experience sometimes questions of diagnosis appear to him to be of rather more than ordinary interest; the result of $x$-ray examination often surprises him; and always he has to adapt his surgical procedures to the material which confronts him. It is around thèse aspects of the question that I propose to speak.

Before saying anything more I want to acknowledge $\mathrm{my}$ personal debt to Sir Berkeley Moynihan (whose work has always been open to visiting surgeons, and whose friendly help has been given to many of us), to $\mathrm{Mr}$. Sherren, and to Mr. William Mayo. To some extent we evolve our individual methods in the course of dealing with our own patients, but certain trails have already been blazed for us, which lessen our anxieties and save our patients from some dangers. To these pioneers our debt is deep.

In dealing with gastric and duodenal ulcers, as with osteo-arthritis, exophthalmic goitre, tuberculosis, and many other diseases, the aim of our profession is to prevent their occurrence, and one by one we may hope to see them disappear; but in the meantime we find our patients seriously ill, being already in a condition which hampers their activities, gives them pain, and puts their lives in danger. Usually they have not consulted a surgeon until their disease has become well established, but many of them have come to you earlier, and some of them reach the surgeon without having had sufficient guidance. Therefore etiology should always be present in our minds. The hygiene of the mouth, arrangement of diet, treatment with drugs, limitation $\mathrm{c} f$ smoking, and proper rest, may cure in the early stages, and will relieve in all. No surgical operation should be undertaken until all these points have been attended to; for by these means operation may sometimes be rendered unnecessary, and operation will fail to achieve its object if tho cause of the condition is not removed.

At what stage, then, are we to say, "This patient should be subjected to operation?" For myself, that time is when the patient, in spite of efficient medical treatment, suffers

* Given at Felixstowe to the Suffolk Branch of the British Medical Association, October 31st, 1924 
so much pain or inconvenience that work cannot be carried on, sleep is interfered with, and life becomes a burden. The symptoms may never have responded to treatment, or may have recurred from time to time in spite of it; or certain complications may occur which force our hands.

The cases which I propose to discuss are all such as have reached the stage at which, in the judgement of physician and surgeon, the time has come for surgical help in treatment. I put it in that way because it is only " surgical help" in treatment. These patients are not all cured by operation in the way a person is cured who has had an appendix removed during an attack of appendicitis, or cholecystectomy performed for a stone impacted in the cystic duct. A great many are cured; others are so greatly improved that, with ordinary care and some guidance, pleasure in life is regained and work can be carried on with zest. $A$ few have had so large a part of their stomachs destroyed by a long-standing callous ulcer that, whatever method of operation may have been adopted, they are still not normal beings afterwards; they will have been relieved of their intense pain, but will always require guidance in feeding. Then, again, occasionally a gastro-jejunal ulcer will follow a gastro-jejunostomy. It is therefore obvious that there should always be the closest co-operation between physician and surgeon if the patient is to derive the benefit he has the right to expect from our profession.

In an uncomplicated case of duodenal ulcer the symptoms are characteristic. Even in the clearest cases it is satisfactory to have a skiagram which demonstrates the lesion. Fig. 1 is from a patient (of Dr. Morison) whose symptoms might have been taken from a textbook, so accurately did they fit in with the recognized picture. He had pain two hours after a meal, more food always " sending it away." He regularly had an attack of acute pain at 3 or 4 o'clock in the morning, which he would relieve by taking an alkaline mixture; he would then go to sleep again. $\mathrm{He}$ had the usual periods of complete freedom from pain. The skiagram shows the distortion of the duodenum, with the barium mixture trickling past the obstruction in a thin stream, and filling the duodenum farther on. But $I$ find that many of the patients, by the time they reach the surgical ward of a hospital, do not give this clearly cut history. In some it can be elicited by careful investigation; in others I fail to obtain it.

Pain such as we expect has been absent in one group of cases. In illustration of this group I show skiagrams of a man and woman, both of whom discovered blood in their stools. From the man I could only get the slightest history of indigestion, and he had not troubled to modify his food as so many patients do; but the melaena had occurred frequently enough to alarm him. The woman stated that she had "queer feelings" two hours after a meal, but her own description of it was that "it was like someone pushing a fist into her back." I could not get an admission from her that she suffered any pain, or that there was abdominal discomfort. Her haemorrhages had been severe. The man was aged 46 and the woman 58. The most common cause of severe melaena is duodenal ulcer; but from the history I could not otherwise satisfy myself of the presence of duodenal ulcer. A skiagram of the man (Fig. 2) gives a cruciform picture from the distortion of the first part of the duodenum. Fig. 3 is a skiagram of the woman, showing the crater of an ulcer filled with barium. In cases of this kind a good skiagram gives conclusive evidence, such as can be obtained in no other way, and it shows the extensive duodenal ulceration that may occur without pain.

As opposed to the cases with unusual freedom from pain, there are others in which pain is atypical in time or intensity, either because of the complications of the lesion itself or of associated conditions. A man was known to drink more wine than he should, and to do so throughout the day. For the last two or three years he had taken considerably too much. During the last eighteen months he had had severe bouts of pain in the upper abdomen with vomiting. It began each time with a feeling that he was not fit, and that an attack was imminent. After a day or two this deepened into pain right across the upper abdomen, which steadily increased in severity and never stopped except when he vomited, which he did several times a day. Vomiting gave relief for an hour or two, the pain then gradually returning. The intense stage of the attack lasted about three days, and then the pain and vomiting subsided. He was closely questioned as to any relationship between the pain and food, but nome could be detected. I believed his attacks to be due to alcoholic gastritis, but I suspected that he might have gall stones, for when the pain was present it was continuous, and when the attack passed his digestion was good. Exploratory operation was performed rather under protest, and a very large duodenal ulcer was found, the inflammatory mass being firmly adherent to the head of the pancreas and the neck of the gall bladder. The liver was cirrhotic. These symptoms were quite unlike those usually present with duodenal ulcer. The patient had been examined by the $x$ rays before I saw him. I believe a skiagram by an efficient radiographer would have definitely demonstrated the lesion.

An ulcer on the free surface of the duodenum may perforate, but this we are not considering to-day. Apart from perforation its base may become adherent to neighbouring structures and surrounded with an inflammatory mass; Fig. 4 illustrates such a case. A narrow channel filled with barium mixture is seen over a considerable length in the pyloric region; the stomach is filled proximally to it, and the duodenum ballooned distally to it, implying the presence of the rigid inflammatory mass which surrounded the duodenal region and showing the barium filling the deep narrow penetrating ulcer. (This skiagram will be referred to more than once later. It presents points of resemblance to gastric carcinoma, also it shows two calcified tuberculous glands above the lesser curvature.) The ulcer; if not on the free surface, may penetrate deeply posteriorly, producing a large inflammatory mass, which may involve, and not infrequently penetrate, the head of the pancreas. Either of these conditions may induce pain which is intense and almost constant, quite unlike the typical pain of duodenal ulcer.

When these conditions have caused narrowing of the lumen of the duodenum, the signs and symptoms of retention of gastric contents are added to those of ulcer. Furthermore, there are sometimes other signs associated with these last mentioned conditions which may be perplexing. I call to mind five men, each of whom had a palpable tumour, seeming to be a little larger than a golfball, morable within limits. A distinguished American surgeon has made the pronouncement that when a tumour of the stomach is big enough to be felt it is too late for surgical cure. All of these men had apparently reached that stage. In each the condition was associated with pain which had become very constant. Whether a lump is due to a neoplasm or an inflammatory mass some stenosis is always present, and in each of these there was evidence of gastric retention. One man had vomited constantly for ten days. Another carried a stomach tube in his pocket, and drew off the stomach contents as soon as the pain became intolerable. A third had vomited so constantly for so long a time that his medical attendants regarded surgical intervention as being quite impossible. In these three patients emaciation was extreme; in the other two vomiting and wasting, though present, were not so marked. Fig. 5 shows a pool of barium mixture lying at the bottom of an enormously distended inert stomach; the barium-filled crater of the duodenal ulcer is seen above and to the left.

It is extremely difficult in the presence of a palpable tumour to come to an exact diagnosis between carcinoma at the pyloric end of the stomach and duodenal ulcer penetrating deeply and surrounded by a mass of inflammatory tissue. The inflammatory condition does not necessarily fix the tumour. The history may be long or short. A long history suggests the possibility of a gastric ulcer which has become malignant, a short one that the lesion is unlikely to be an ulcer and has been a carcinoma from the first.

Does $x$-ray examination help us in these cases? Some patients are far too ill for investigation to be made at the time when the surgeon first sees them. Two of these five 


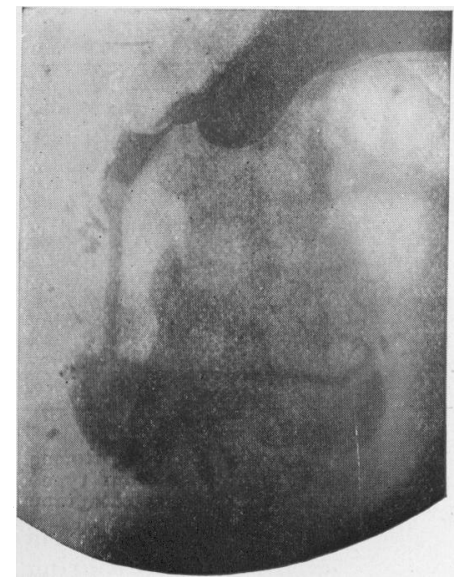

Fig. 1.-Stenosis in first part of duodenum. (Skiagram by Dr. Vilvandre.)

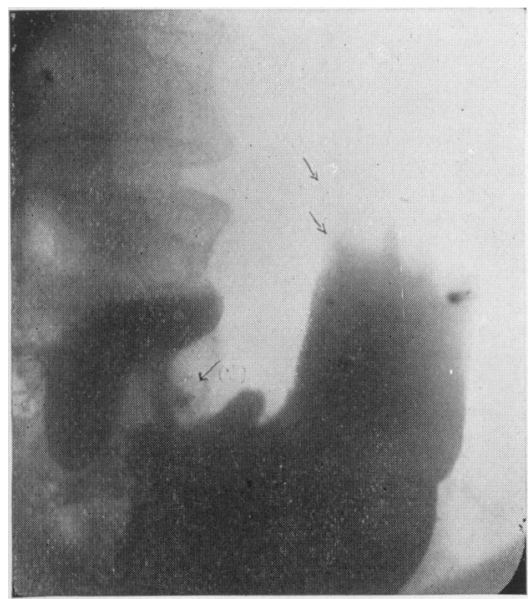

FiG. 4.-Duodenal ulcer; stenosis. Crater flled with barium emulsion. Ballooning of duodenum beyond stricture; indentation of pyloric end of greater curvature simulating flling defect of cancer. Two calcified tuberture. (Skiagram by Dr. Vilvandre.)

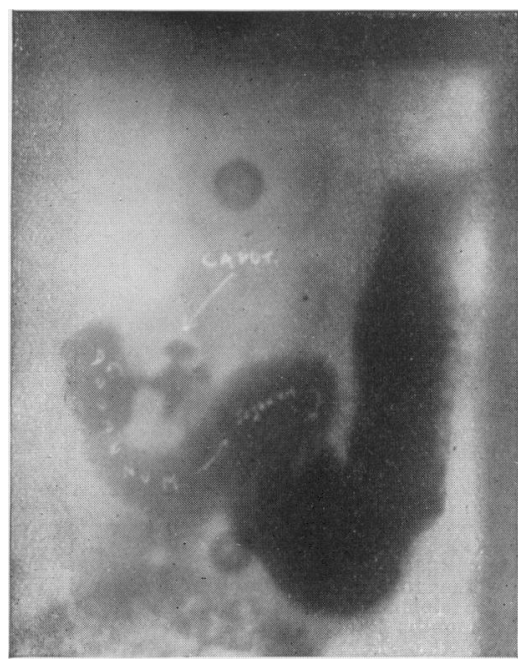
FIG. 2.-Duodenal ulcer; a not infrequent type of distortion with ballooning beyo
(St. Bartholomew's Hospital skiagram.)

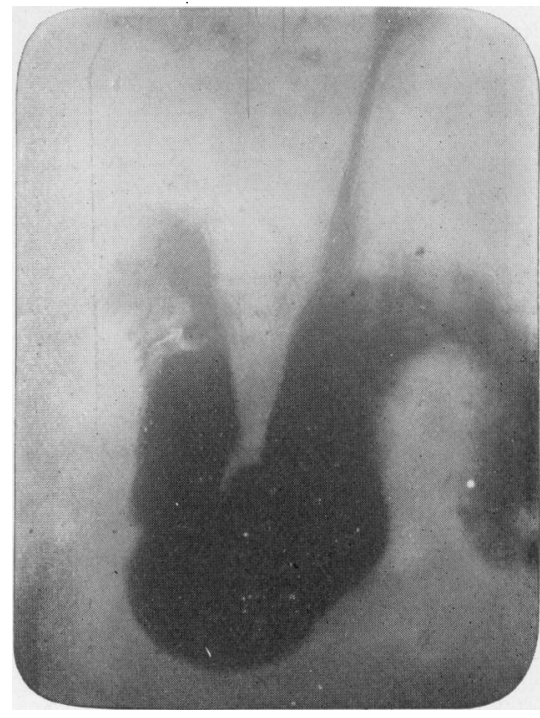

Fig. 3.-Duodenal ulcer. Crater filled with barium mixture. (St. Bartholomew's Hospital skiagram.)

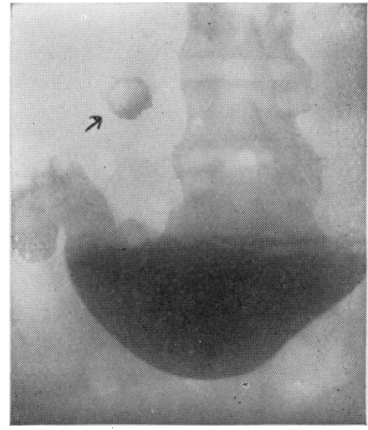

Fig. 5.-Large circular ulcer of duodenum filled with barium mixture. Pool of barium mixture lying in a big atonic (St. Bartholomew's Hospital skiagram.)

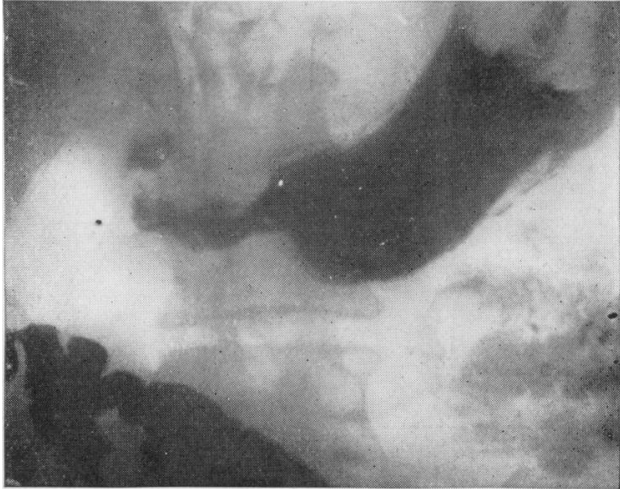

Fig. 6.-Carcinoma of pyloric segment of stomach, showing filling defect. (Skiagram by Dr. Vilvandre.)

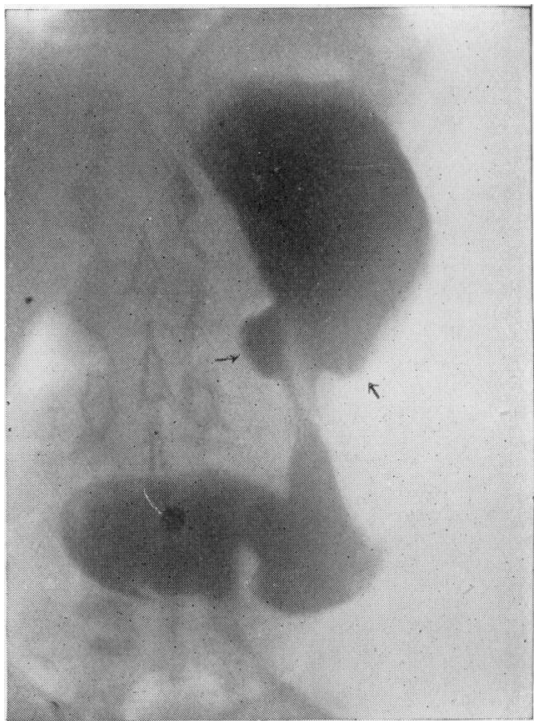

FIG. 8.-Hour-glass stomach with saddle-shaped gastric ulcer : upper part invading left lobe of liver; lower part invading pancreas. Sleeve resection. Patient perfectly well. (St. Bar-
tholomew's Hospital skiagram.)
Fig. 7.-Marked stenosis of commencement of the duodenum due to ulcer, associated with great dilatation of the stomach and emaciation; death from bronchopneumonia following opera-

tion. (Photograph by Miss Vaughan.)

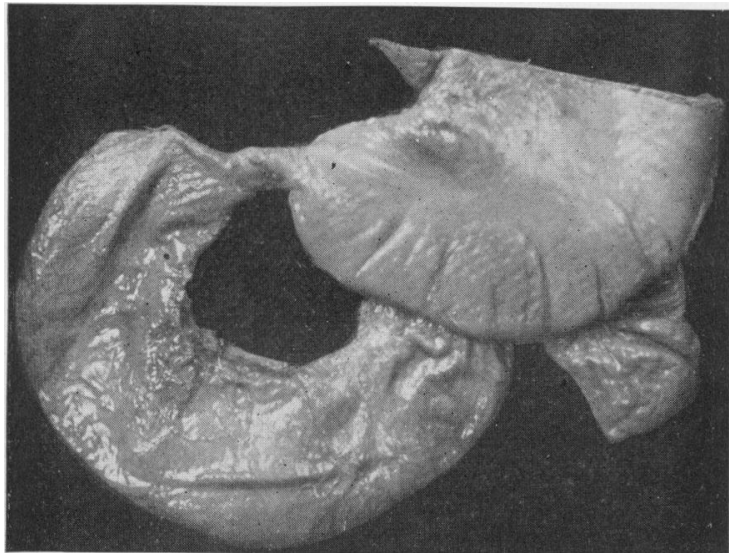




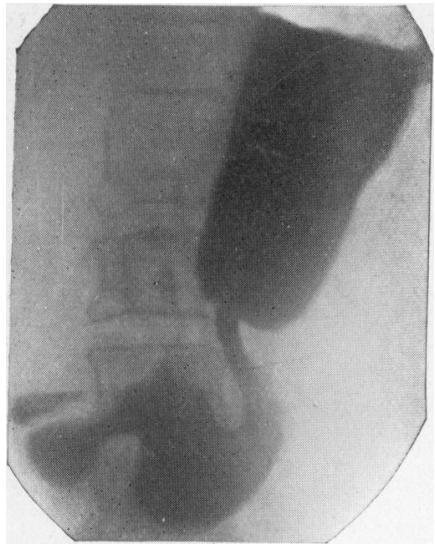

Fig. 9.-Very narrow hour-glass Fig. 10. (St. Bartholomew's Hospital sliiagram.)

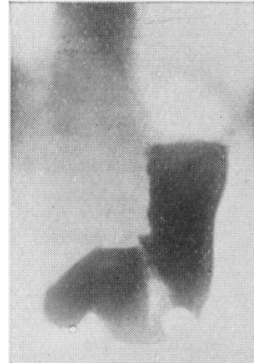

(a)

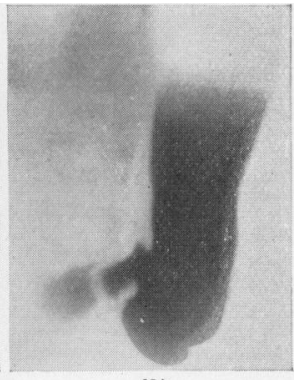

(b)

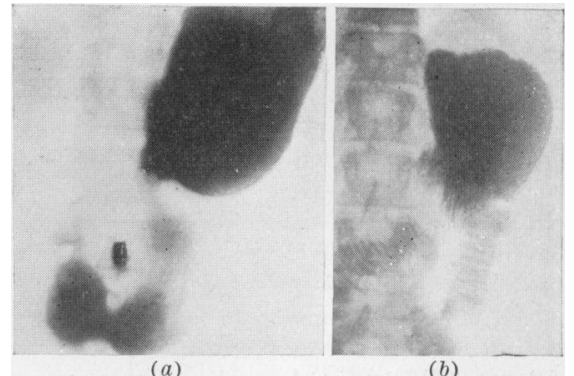

FIG. 11.-(a) Hour-glass constriction of stomach. (b) The stomach after the Polya-Balfour type of operation. (St. Bartholomew's Hospital skiagrams.)
FIG. 10.-(a) The same patient as Fig. 9 one month after operation, showing filling defect caused by the inroling of the approximated (b) Twelve months later; the filling defect has disappeared, the shape and motility of the stomach are practically normal.

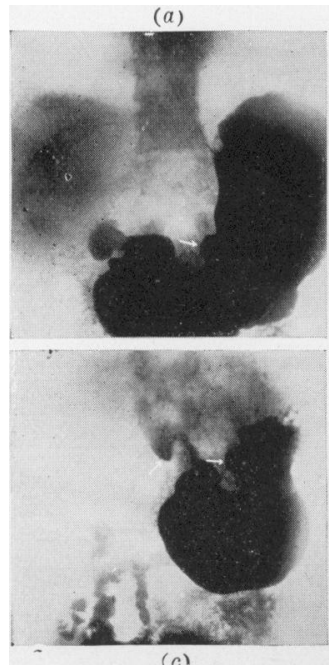

(c)

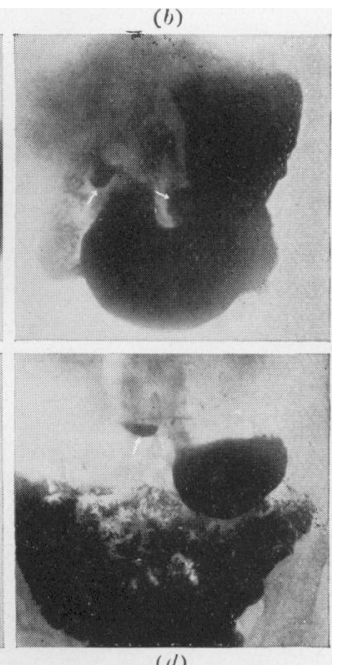

(d)

FIG. 12.-(a) Immediately after a meal. The gastric ulcer is shown at the middle of the lesser curvature. Notice also the barium entering the cardia. (b) Two hours later; the gastric and duodenal ulcers are both shown. (c) The same patient after six hours. Both ulcers are shown. (d) At eight hours after the meal. The duodenal ulceinin in the able residue remining in the stomach. The pastric ulcer malignant. (Skiagrams by Dr. Salmond.)

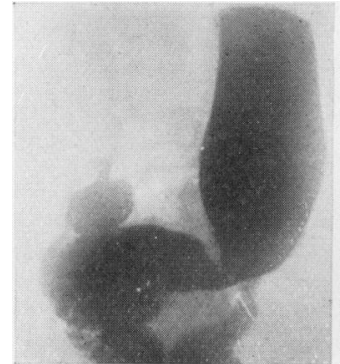

(a)

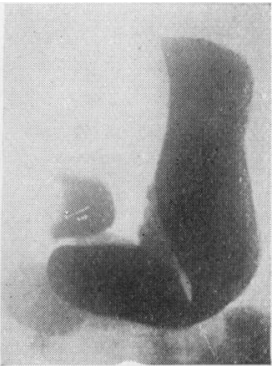

(b)
FIG. 13.-(a) Deep incisura extending from lesser curvature, showing narrowing of stomach at the site of a healed ulcer which had been active thirty years previously. (b) Skiagram taken three months
after $(a)$. The gall bladder contained calculi. (Skiagrams by Dr. Salmond.)

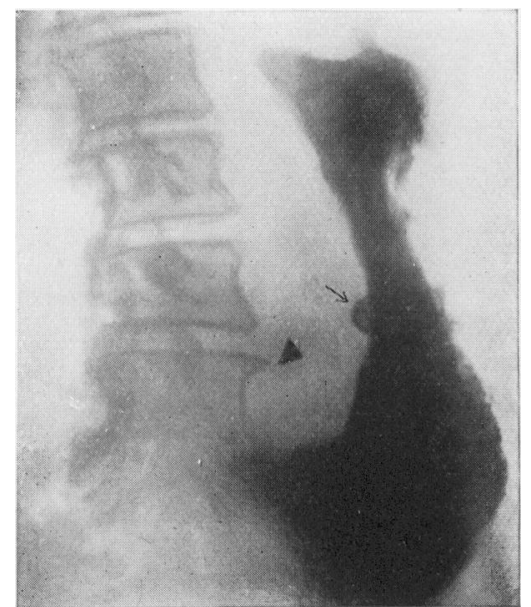

FIG. 14.-An ulcer below and behind the lesser curvature. With a thick meal it is only seen on rotating the patient almost transversely. (St. Bartholomew's Hospital skiagram.)

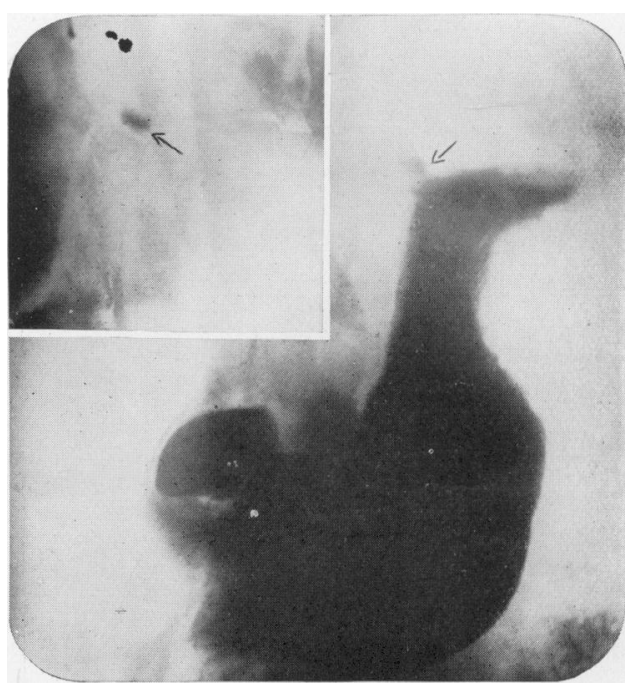

FIG. 15.- ? Ulcerated diverticulum at cardiac orifice of stomach in a tuberculous patient. Inset: Diverticulum still filled with barium mixture after stomach is practically empty. (New Lodge Clinic skiagrams.) 
had been steadily getting worse until the operation had to be performed as an emergency measure. If the patient is not too ill, $x$-ray examination may settle the diagnosis. Fig. 5 is obviously the picture of a large duodenal ulcer with such degree of associated stenosis that the peristaltic vigour of the stomach had diminished and atony resulted. But if Fig. 4 be compared with Fig. 6 it will be seen that the problem is more difficult. The history was identical in each of these two cases, and the age practically the sameabout 60 years. In each a tumour could be felt of the same size. Each moved with respiration and was mobile apart from respiration. In each the skiagram appeared to show a filling defect at the pyloric end of the stomach; the indentation of the wall in this area and the narrowing of the pyloric region over a considerable length may be noted. From the skiagram $I$ believed the condition in Fig. 4 to be carcinoma, and I was sure that Fig. 6 indicated the same. Both patients were too ill for a complete operation to be performed at one time. In each gastro-jejunostomy was performed in order to tide the patient over the emergency. In each the tumour was seen and palpated, and in each it appeared to be carcinoma. In one a carcinoma was removed a fortnight later. In the other the second operation was delayed on account of lung complications, and when the abdomen was afterwards opened for the partial gastrectomy all signs of the tumour had disappeared, the pylorus and duodenum being then soft and unscarred. (The deeply penetrating ulcer shown in Fig. 4 had been on the posterior surface.)

It is easy to be wise after the event. In a review of these cases it should be realized that in Fig. 4 the barium entered a long narrow ulcer cavity, quite unlike the cavity of a carcinomatous ulcer; the inflammatory mass surrounding this had thickened, hardened, and indented the stomach wall in its vicinity, and given a skiagram bearing a considerable resemblance to that produced by the carcinoma in Fig. 6.

An associated pathological condition will alter the characteristic history of duodenal ulcer. We have all seen a gastric and duodenal ulcer in the same patient. Moynihan ${ }^{1}$ states that a duodenal ulcer is present in 12.3 per cent. of all cases of gastric ulcer. Tuberculous glands are also not infrequently found. Fig. 4 shows, in addition to the duodenal ulcer, two calcified glands along the line of the lesser curvature of the stomach. These may not only make the history less clear, but make the prognosis less perfect. Needless to say, when an abdomen is opened the search for other pathological conditions should be very thorough, even though the ulcer is obvious; for if tuberculous glands are present-as they have been in several of my cases-and are not discovered, the surgical treatment may be deemed to have failed, while actually the remaining symptoms are due to other causes.

In at least two conditions special care is required in planning surgical interference. These are: (1) extreme emaciation from increasing pyloric stenosis with consequent starvation, and (2) repeated haemorrhages. On four occasions I have been able to perform a gastro-jejunostomy very easily and quite successfully with local anaesthesia, when the patient appeared to be too emaciated and weak to justify the use of general anaesthesia; on one occasion I lost, through lung complications, a patient who I believe would have been saved if I had used local anaesthesia. He was the patient who had either vomited or emptied his stomach with a tube every day for many months in order to relieve his pain, and he was emaciated to a skeleton. Fig. 7 is a photograph of the organs removed at autopsy. The first part of the duodenum is seen to be narrowed. It is very thick-walled and the lumen is almost obliterated. Sometimes a patient recovers from repeated haemorrhages sufficiently to undergo operations safely, but occasionally it seems impossible to control the haemorrhage from the ulcer, and day by day the patient becomes weaker and paler. These patients have several times been rendered fit for the appropriate surgical measures by means of blood transfusion.

In gastric as in duodenal ulcer there are long periods of latency, which lead patient and practitioner to believe that the pathological condition has disappeared. There may be some cases in which the ulcer has healed, but there are many in which it has not. Again, as in duodenal ulcer, the history of gastric ulcer in its early stages is much more characteristic than in the later stages. In many cases which come to the surgical wards of a general hospital the characteristic history has long passed, and the story may have to be reconstructed from the patient's memories of long ago. One man recently came to us who twenty years before had had the orthodox symptoms-pain an hour and a half after food, lasting half an hour, disappearing before the next meal. He had continued in this state for fifteen years, being sometimes better, sometimes worse. Then a severo haemorrhage rendered his admission to hospital necessary. He was discharged " cured." Still later-twenty years after the onset of the first symptoms-there was another attack of haemorrhage, and from this time onwards he was scarcely ever free from pain, day or night. The pain, the sleeplessness which the pain induced, the inability to take food, the loss of blood (which could always be detected in his stools), and the resulting exhaustion made him pallid, emaciated, and very ill. During all this time the ulceration had been slowly progressing; the base of the ulcer became adherent to and eroded the body of the pancreas, while surrounding inflammation in the wall of the stomach resulted in the formation of an hour-glass stomach.

When once these ulcers havo penetrated the gastric wall so that the base is formed by structures outside the stomach, the reaction surrounding them may be intense, and the inflammatory products constitute a mass which may fill the upper left quadrant of the abdomen, and extend below tho umbilicus. This area will be rigid and without movement on respiration. The rigidity and the tenderness may prevent any effective examination of the abdomen. In ono patient of this type pain occurred within a few minutes of taking food and lasted about two hours. He would vomit at any time during this period; but obtained only slight relief. The pain was violent; it "doubled him up," and prevented him from sleeping; he was afraid to take any food except egg and milk. Ho obtained a little relief by lying in the left latero-prone position. Four weeks before admission to hospital he had romited blood. He was far too ill for an $x$-ray examination to be made. He presented the picture of late malignant disease of the stomach, but becauso of his age (34) his abdomen was explored. There were enlarged glands in the great and lesser omenta. A largo hard mass involved the lesser curvature and posterior wall of the stomach and extended up to the cardia; it appeared to be infiltrating the left lobe of the liver. A partial décollement was made in order to examine the stomach from below; it was found to be firmly fixed to the pancreas. The condition was regarded as malignant and inoperable. Three glands were removed for examination and the abdomen closed. The glands were found to be non-malignant, and the patient improved sufficiently to enable an $x$-ray examination to be made. The result is shown in Fig. 8 . The barium mixture is seen filling a large ulcer crater above the lesser curvature; the base of this was formed by the liver, and opposite this, bulging below and overlapped by the greater curvature, is seen the edge of a very large barium-filled crater which had burrowed into the pancreas. The middle third of the stomach immediately below these ulcers was almost obliterated. When the stomach was dissected off the liver and pancreas scarcely any middle third remained. The free edges of the proximal third were very ragged and the induration along the lesse: curvature and posterior wall extended almost to the cardiac orifice. The patient suffered from marked aortic disease, and as the distal third of the stomach was healthy it seemed wise to save time by trimming off the ragged indurated edges and joining up the two ends of the stomach. This patient is quite well two and a half years after the operation.

Even when patients are not so ill as in the last case the diagnosis is not always easy. In women it has not infrequently happened that the pains have been so mucil worse about the menstrual periods, and have appeared not to be so definitely related to food, that attention has beell directed to other organs of the body. Of two women who came for treatment, one had had four, and the other three, 
previous operations on the lower abdomen and pelvic organs; all these operations were for pain, which was ultimately proved to have been due to gastric ulcer. In one of them the skiagram shows an extremely narrow hour-glass constriction; this case is interesting in that the ulcer perforated immediately above the site of constriction. The hole was big enough to admit a finger, and the stomach contents were mixed up around and between the adhesions consequent on three previous abdominal operations (one of which unfortunately had been a double oöphorectomy). The perforation was closed, and, as soon as the patient was fit for it, an $x$-ray examination was made. The result is shown in Fig. 9. It was realized that this patient would be in a precarious state if the stomach were left in this condition. It was known that the abdomen was matted with adhesions rendering it difficult to apply the jejunum to the proximal end of the stomach, so it was decided to perform a sleeve resection. The resulting shape of the stomach soon after operation is shown in Fig. 10a. At this early stage the filling defect in the stomach outline caused by the inrolling of the approximated edges of the stomach wall by Lembert sutures is seen. The stomach functions well, and the patient has since married. A recent photograph has been obtained showing the stomach shape and motility to be practically normal (Fig. 10b).

It is not often possible to apply stomach clamps in the manner figured in textbook illustrations of sleeve resection. The cases in which clamps can be applied rarely require sleeve resection, and in those which demand it the surgeon must use all the skill of which he is possessed to cobble together the distal third on to the ragged remains of the proximal piece, so that the posterior wall may be safe and efficient. In the patients upon whom it has been found necessary to perform sleeve resection on account of the exigencies of the condition the late progress has been quite good. Fig. $10 b$ shows a stomach in which abnormality can scarcely be detected.

Usually in cases of hour-glass stomach due to ulcer in the middle third, when the case has been carefully worked out, and the $x$-ray examination-fixing the site of the ulcer and the degree of mobility of the stomach-has enabled the operation to be properly planned before the abdomen is opened, a partial gastrectomy is performed, the proximal end of the duodenum is closed, and the jejunum anastomosed with the open end of the remaining piece of stomachBalfour's modification of the operation originally planned by Polya. Figs. $11 a$ and $11 b$ show the hour-glass constriction, and the condition of the viscera subsequent to operation. This operation has proved to be most satisfactory in the cases which have been severe enough to necessitate it. I believe the patients are more free from subsequent discomforts than after any other operation for gastric ulcer in this situation. The only disadvantage I have found is that in a few cases the size of the meal has to be restricted. If this is regarded the patients are happy, and as this operation has usually been performed for very disabling pain they do not mind the slight restrictions. Some are so hungry afterwards that, as one man put it, "I really cannot keep myself away from food."

I have already mentioned the simultaneous occurrence of gastric and duodenal ulcer. Figs. $12 a, b, c, d$, show the appearance on $x$-ray examination of such a dual condition: $12: \imath$ is immediately after the meal; $12 b$ in two hours; $12 c$ shows the condition almost unchanged in six hours; and at eight hours $(12 d)$ there is still a barium residue in the crater of the duodenal ulcer. At operation the gastric ulcer was found to be about one and a half inches in diameter and an incin in depth. Examination of the specimen showed that it had penetrated into the body of the pancreas. The duodenal ulcer was on the posterior inferior aspect of the first part of the duodenum; that also is a considerable ulcer. A finger could invaginate the anterior wall of the viscus into the crater of each ulcer easily. The skiagram $12 a$ shows barium in the oesophagus and entering the stomach, so that the position of the cardia is defined. It also shows that there is probably sufficient of the proximal part of the stomach still unaffected to permit a partial gastrectomy. The Polya type of operation, planned to include both ulcers, appeared to be the most suitable pro- cedure for the condition, and it was performed. The concave floor of the gastric ulcer consisted of pancreas. It was wiped clean, and a layer of great omentum tacked over it with a catgut stitch after the stomach had been dissected free from it. When this man was seen by his latest consulting physician before operation he stated that ho had no pain in his abdomen, but an intense pain deep in the middle of the right side of his chest and in his right shoulder-blade, and that it had no relation to food. It had become constant and unbearable. Microscopical examination of the gastric ulcer showed that it had become malignant, thus bearing out the statement that gastric ulcers more than one inch in diameter are prone to be malignant.

Figs. $13 a$ and $13 b$ are shown to illustrate the permanent change which may occur in the shape of a stomach with the healing of a chronic ulcer. These two skiagrams were taken with an interval of three months between them. A similar picture is given on each occasion-a deep narrow incisura cutting into the stomach from the lesser curvature. This was unaffected by peristaltic waves. On other films of this patient the outline of the gall bladder can be easily seen. Thirty years previously he had suffered from a gastric ulcer. He had been seriously ill for six months and an invalid for two years. After four years he could take ordinary food, but often suffered from indigestion. Twelve months before these skiagrams were taken discomfort after food became more insistent, but the symptoms were rather suggestive of gall stones. At operation the gall bladder was found to contain many gall stones. After dealing with this, and removing a firmly bound down appendix, the stomach was opened in its long axis across the constriction for two and a half inches. This was to determine if there was any active ulceration present, and to restore a normal shape by suturing the incision transversely to the long axis of the stomach. The ulcer had healed, the stomach wall was soft, but the stenosis was such that it would just about admit a thumb.

Fig. 14 is the skiagram of an ulcer which, in the days when a large barium meal was given, instead of the small fluid meal, might only be discovered by a lateral view of the patient. The position of the vertebrae shows how much this patient was rotated before the ulcer became visible.

Two conditions are met with which present great difficulty: one is persistent uncontrollable haemorrhage from an ulcer; the other is gastro-jejunal ulcer. The records of St. Bartholomew's Hospital show that during the years 1921 to 1924 inclusive seventeen patients died from haemorrhage attributed to gastric or duodenal ulcer. Blood transfusion has made it possible to deal with these cases under conditions which would have been impossible before the days of transfusion. For this condition some surgeons perform partial gastrectomy. It has seemed to me, however, that a less severe operation is wiser in patients who are almost moribund; and in the cases which have come under my care partial gastrectomy would scarcely have been effective. In one there was a duodenal ulcer in the distal half of the second part of the duodenum, and on the posterior surface. On opening the duodenum a large callous ulcer was evident, and blood was seen trickling from under its upper margin. It was possible to underrun it with fine catgut and the bleeding was controlled at once. Gastro-jejunostomy was then performed. Blood transfusion was carried on by $\mathrm{Mr}$. Keynes during the operation. Bleeding never recurred, and the duodenal ulcer healed, for $I$ saw it at a subsequent operation. A gastro-jejunal ulcer supervened in the following year, and of this I will speak later.

In one case of persistent haemorrhage from a gastrio ulcer the ulcer was so high that on opening the stomach and emptying it by suction it was impossible to reach and underrun the spot where the oozing was readily seen. It was reached by a long-handled cautery at a dull red heat and the bleeding was seen to stop. A gastro-jejunostomy was performed to prevent gastric retention and allow alkaline intestinal fluid to enter the stomach. I am not persuaded that either of these reasons is physiologically sound, but the patient-who for a month had been incapacitated and who at the time of the operation was moribund-has been working as a stevedore ever since. 
Haematemesis due to acute ulcer seldom requires operative interference. Rest, medical treatment, and, if necessary, blood transfusion, has, in our experience, been sufficient to arrest the condition and recovery has followed; but with a chronic ulcer it is often otherwise. In spite of rest and blood transfusion the haemorrhages may continue with fatal result. In an increasing number of this class of patient we have been able to operate with success.

I have seen one case of syphilitic ulceration-a patient under the care of Dr. J. H. Drysdale. The patient suffered from gastric symptoms, and $x$-ray examination revealed the presence of a gastric ulcer. The Wassermann test was positive, and the symptoms cleared up with appropriate treatment.

In another case the diagnosis is not yet absolutely settled. In Fig. 15 a sacculus opening into the stomach close to the cardiac orifice is shown filled with barium emulsion. Inset is shown the cavity still filled with barium after the stomach had emptied. Severe haematemesis had occurred, presumably from this, and I had been asked to take in the patient. Dr. Hurst believes the condition to be ulceration in a diverticulum of the stomach. The patient was subsequently found to be suffering from phthisis-from which she is recovering so that further opportunity for $x$-ray examination will probably be possible.

Five cases of gastro-jejunal ulcer have come under my care. Of these, one was the patient mentioned above, upon whom I operated after repeated severe duodenal haemorrhages extending over a month and increasing in severity. He was therefore very much debilitated when the operation was performed, and recovery was slow; whether this influenced the formation of the gastro-jejunal ulcer I am not prepared to say. Another case followed an operation which I performed for a perforated duodenal ulcer. The perforation was closed and a posterior gastro-jejunostomy made. Two years later an ulcer at the site of the stoma perforated. As far as I know these are the only two which have followed my own operations. In one other case I found strands of infected silk hanging into the lumen of the stoma from several ulcerated areas, and the whole circumference was thickened by the infection and granulation tissue surrounding the buried part of the silk. Operation for this condition is very difficult. There is always an inflammatory mass in the mesocolon surrounding the base of the ulcer. Often it is closely adherent to the colon, and separation of this is never easy. The stoma is freed from the mesocolon and the jejunum separated from the stomach; the opening in the jejunum may be closed and some variation of th Polya operation performed, or the jejunum may be divided and the operation completed as described by Sir Berkeley Moynihan.

$X$-ray examination with a barium meal has become a valuable procedure. Some years ago we were glad when positive evidence was obtained, but we did not expect too much from the method. If the surgeon and radiologist work together, evidence is obtained by a screen examination which is not always recorded on films or plates, and operation is now not often performed if there is no $x$-ray evidence of ulcer. Some patients are too ill for the examination to be made. If it is done by an unskilled radiologist the findings may be worse than useless, because inaccurate evidence is actually misleading. If ulceration is present, distortion of the outline of the viscus is sometimes seen (Figs. 1 and 2); a deposit of barium may remain in an ulcer crater (Figs. 5 and $13 b$ ). $X$-ray examination not only establishes the diagnosis, but also shows the position of the lesion, and knowledge is obtained of how much of the viscus appears to be normal, so that the type of operation may be considered, and perhaps definitely planned before the abdomen is opened. If a dual condition is present, as in Fig. 4 (duodenal ulcer with tuberculous glands) or Fig. 12 (gastric and duodenal ulcer), the surgeon is forewarned.

Of these skiagrams some have been reduced to a size appropriate for publication by Miss Vaughan in the Dunn Laboratories of the Professorial Units at St. Bartholomew's Hospital, and some by Kodak, Limited.

\section{A COMPARISON OF DRIED, EVAPORATED, AND} FRESH COW'S MILK : AN EXPERIMENTAL STUDY.

\author{
BY
}

GLADYS ANNIE HARTWELL, D.Sc.

(From the Physiological Laboratory, Household and Social Science Department, King's College for Women, Kensington, London.)

Ar the present time there are many varieties of dried, evaporated, and condensed milks on the market. As it seemed possible that there might be fundamental differences between various sorts of dried and evaporated milks, a series of feeding experiments on animals have been made.

Sherman, Rowe, Allen, and Woods, ${ }^{i}$ experimenting with rats, observed that "with bread and milk there was continued growth at a normal rate to full adult size." It seemed possible, therefore, to compare the different kinds of evaporated milk by feeding rats on each kind of milk and bread as the sole diet. According to a report of the Medical Research Council (1924) on the presence of vitanins in different varieties of milk, vitamins $\mathbf{A}$ and $\mathbf{B}$ are quite well represented, but vitamin $\mathrm{C}$ may be absent, or only traces present, in evaporated (condensed unsweetened) and dried milks.

Simmonds ${ }^{2}$ concluded that the rat does not need the antiscorbutic factor for normal development, and that a diet of milk and bread might be expected to contain all the essential constituents of a complete diet for this animal. The experiments here described were started when the rats were just weaned (three weeks old), and were continued for twenty-two weeks, during which time several litters were born and reared. I regard it as a more searching test of the adequacy of a diet if gestation and lactation are included, and the experiment made not merely for the actual growth period. Mitchell, ${ }^{3}$ in his article on the nutritive value of proteins, pointed out that the demands of growth, gestation, and lactation are all separate problems as far as the biological value of protein is concerned, and it is highly probable that this applies equally to all food factors.

The experiments were made on the following lines:

(a) Animals and Method of Experiment

Rats were used in these experiments. They were all bred in the laboratory 4 and were all in equally good condition at the beginning of the experiment. Twelve animals, six males and six females, were fed on each diet. They were kept in a large cage together so that breeding could take place as soon as the rats reached so that breeding could take place as soon as the rats reachea
maturity. Each animal was weighed every day (except Saturday and Sunday) for the first few weeks, and after that, when growth was less rapid, only once a week: They were fed once a day. The females were removed to separate cages before the birth of the young. As far as possible, the method we adopt is to remove a doe from the large cage two or three days before the birth of the litter. She suckles her young for twenty-one days, after which she is returned to the large cage. If the young do not survive (as may happen when the diet is poor) the doe is returned to the large cage again when all - her young have died. Rats have large litters, and our custom is to reduce the number of young to six on the second day, in order to prevent any undue strain on the mother rat, and to make the growth curves of the young comparable. The combined weight of even six babies towards the end of lactation may exceed that of the mother rat, but on a suitable diet she is capable of rearing her offspring without suitable diet she is capable of rearing her offspring without
detriment to herself. From the second day onwards, the motlier and her young are weighed daily; she is given ample food, all constituents of the diet being well mixed in the form of a paste.

(b) Foods.

1. White bread bought from a baker was used, no crust being giren. The bread was weighed one day old

2. Milks. - The fresh cow's milk used was of good quality, was delivered in a bottle, and on standing always showed a large amount of cream. It was heated to $70^{\circ} \mathrm{C}$. before being given to the animals. Of dried milks a well known brand (referred to as sample $A$ in a previous paper ${ }^{5}$ was chosen as being a variety largely used by the general pablic. The brand of evaporated, non-sweetened milk chosen was selected because it appeared to be a popular article.

3 . proportions.-The evaporated milk was diluted accorling to the directions on the tin, and the specific gravity of this reconstructed food was approximately that of fresh cow's milk. With the dried milk 12 grams of solid were mixed with $100 \mathrm{c.cm}$. of water. This is approximately the amount of solid required to imitate $100 \mathrm{c.cm}$. of whole milk. My object was to use suck amounts as would be used by the general public, following the directions given on the tin. Thus, when told to measure ond 